\title{
Das Grunddilemma und die Natur des Staatsnotstandes
}

\author{
Eine deutsche Problematik mit ausländischen Augen
}

Wenn ein ausländischer Jurist die deutsche verfassungsrechtliche Literatur studiert, merkt er schnell und überrascht eine Eigenartigkeit dieser Wissenschaft: die anderswo unüblich starke (wenn auch in der letzten Zeit abgeschwächte) Konzentration auf einen (im wortwörtlichen Sinne) Randbereich des Verfassungsrechts, auf das Staatsnotstandsrecht. ${ }^{\mathrm{I}}$ Die Problematik ist natürlich auch in anderen Ländern bekannt, aber die Leidenschaft und der theoretische Aufwand, mit der dieser Marginalbereich behandelt wird, ist einzigartig.

Dieses Desinteresse anderer Verfassungsrechtswissenschaften und die in der letzten Zeit zu sehende Abschwächung des deutschen Interesses können sich allerdings ändern, und der Marginalbereich kann zu einer höchst aktuellen Problematik werden: Die Terrorismusbekämpfung wirft nämlich Fragen auf, die eventuell mit den in der deutschen Rechtswissenschaft verfeinert ausgearbeiteten Argumentationsmustern des Notstandsrechtes adäquat diskutiert werden können. Diese Fragen betreffen den Sinn positivrechtlicher Regelungen in extremen Situationen, und führen letztendlich zum selben Grunddilemma, wenn auch unter anderer Terminologie. In diesem Sinne könnte man die rechtliche Problematik der Terrorismusbekämpfung als »maskiertes Notstandsrecht« betrachten. Die verschiedenen notstandsrechtlichen Argumentationsmuster wurden zwar bisher nicht direkt in den hochaktuellen juristischen Diskurs der Terrorismusbekämpfung übersetzt (das wird auch hier nicht versucht), aber sie würden sich dazu anbieten. Dies verleiht dem Thema eine »potenzielle Aktualität - wenn auch der klassische Staatsnotstand selbst heutzutage nicht mehr zu den aktuellsten Themen gehört.

Dieser Artikel möchte durch die Darstellung der bisherigen Ergebnisse der deutschen Literatur und des positiven deutschen Verfassungsrechts ein paar gefährliche und inkonsequente Sackgassen der juristischen Behandlung des Staatsnotstandes hervorheben, und somit als Ausgangspunkt für eine eventuell weiterführende Diskussion im obengenannten Sinne dienen.

\section{Das Grunddilemma des Notstandsrechtes, gezeigt anhand von typischen Regelungsgehalten}

Der Verfassungsstaat kann in solche staats- bzw. verfassungsgefährdenden Situationen geraten, die mit den Bindungen der Verfassung nicht - oder zumindest nicht in der gebotenen Eile ${ }^{2}-$ zu bewältigen sind (Staatsnotstand). 3 Deshalb müssen diesbezügliche Regelungen (Notstandsrecht oder Staatsnotstandsrecht) ter Frankenberg, Alexandra Hilal Guhr, Rainer Grote, Ute Mager, László Sólyom und Markus Wagner.

2 Zum Zeitfaktor (d. h. Dringlichkeit) diesbezüglich s. C. Möllers, Staat als Argument, 2000, 266.

3 Dies ist von der Verfassungsstörung zu unterscheiden. Bei letzterer geht es nämlich um eine Situation, in der die Störung aus der inneren Lebenssphäre des Staates stammt (z. B. Selbstausschaltung des parlamentarischen Gesetzgebers in den letzten Jahren der Weimarer Republik), s. J. Heckel, Diktatur, Notverordnungsrecht, Staatsnotstand, AöR 1932, 3,10. In diegero. Aufsatz wird nur der Ausnahmezustand, nicht aber die Problematik der Verfassungsstörung ( $z$. B. Gesetzgebungsinotstand) analysiert. 
getroffen werden, die einerseits von diesen Bindungen befreien (Effektivität), andererseits aber Vorkehrungen gegen den Missbrauch solcher Entbindungen enthalten. ${ }^{4}$ Das Grunddilemma des Notstandsrechtes ist die Frage, wie das Gleichgewicht zwischen diesen zwei Anforderungen zu halten ist.5 Das eigentliche Ziel des Notstandsrechtes sollte nämlich sein, den Rückweg in den »normalen "Verfassungszustand zu sichern. ${ }^{6}$ Eine grundlegende, aber oft übersehene Vorfrage dieses Dilemmas ist, wie man mit dem Moment »Gefährdung « umgeht. Einerseits sollen dabei die Schutzgüter bestimmt werden (nicht jede Detailregelung der Verfassung benötigt zum Schutz einen Staatsnotstand - wenn auch die Verletzung üblich und anders nicht aufhebbar ist -, sondern nur ihre Grundprinzipien und die Selbstschutzmechanismen), andererseits soll es klar sein, dass die Realisierung der Gefährdung eine bestimmte Wahrscheinlichkeit erreichen soll. Die Gefahr eines unerwarteten Krieges oder eines katastrophalen Terroranschlages ist immer größer als null - dies heißt aber nicht, dass sich jeder Staat immer im Staatsnotstand befinden sollte.

Um die Dilemma-Problematik näher zu entfalten, werden im Folgenden die Entbindungen und die Vorkehrungen gegen ihren Missbrauch anhand von drei formal-verfassungsrechtlichen Notstandsrechten rechtsvergleichend dargestellt: Ungarn, die BRD und die Weimarer Republik. Der Einbezug von Weimar ist unausweichlich, da hinter den geltenden deutschen Notstandsregelungen wie auch hinter den Literaturmeinungen die geschichtliche Erfahrung von Weimar immer wieder auftaucht, und die heutige Lage (als bewusster Kontrapunkt zu Weimar) ohne dies nicht verständlich erklärt werden kann. Der Einbezug Ungarns ist dagegen damit zu begründen, dass dort die notstandsrechtliche Regelung ähnlich detailliert und ähnlich strukturiert ist wie in Deutschland (von solchen Verfassungen gibt es nicht viele in der Welt), aber teilweise andere Regelungstechniken benutzt werden. Eine völlig andere Lösung (z. B. England oder Frankreich) schien als Vergleichsgrundlage deshalb nicht fruchtbar, weil der Vergleich wegen des grundsätzlich anderen Charakters immer nur auf die Aussage "grundlegend anders « hinauslaufen würde, ohne den Sinn der Einzelregelungen des Grundgesetzes mit einem ähnlichen, aber dennoch unterschiedlichen Ansatz beleuchten zu können.

Das Ziel des Vergleichs ist nicht die Vollständigkeit, sondern das Aufzeigen des Grunddilemmas. Die typischen Regelungsinhalte werden immer unter Berücksichtigung der zwei genannten Gesichtspunkte (Effektivität vs. Gefahren für die Verfassungsstaatlichkeit) analysiert. ${ }^{8}$

\section{I.I Anfang, Ende und Stufen des Staatsnotstandes}

I. Da das Notstandsrecht ein gefährliches rechtliches Instrumentarium aktiviert (d. h. man kann es leicht missbrauchen), ist es angebracht, nicht nur seinen Tat-

4 F. Koja, Allgemeine Staatslehre, I993, 397.

5 P. Kirchhof, Die Zulässigkeit des Einsatzes staatlicher Gewalt in Ausnahmesituationen, in: Deutsche Sektion der Juristenkommission (Hrsg.), Die Zulässigkeit des Einsatzes staatlicher Gewalt in Ausnahmesituationen, I 976,84 .

6 K. Hesse, Staatsnotstand und Staatsnotrecht, in: Staatslexikon der Görres-Gesellschaft, I989, 7. Aufl., V. Band, 202; ders., Grundzüge des Verfassungsrechts der BRD, 20. Aufl. I999, 303.

7 »Die Geschichte ist voll mit solchen Kriegen, von denen jeder wusste, dass sie nie ausbrechen werden. « (ungarisches Sprichwort).

8 Mit der Begrifflichkeit von K. Stern, Das Staatsrecht der BRD, Band II, I980, I 303: »Normativität « vs. »Effizienz«. 
bestand präzise zu bestimmen (Art. i I sa Abs. I Satz I und Art. 9I Abs. I Grundgesetz; Art. I9 Abs. 3 lit. h und i ungVerf; weniger präzis: Art. 48 Abs. 2 Satz I WRV), ${ }^{9}$ sondern auch seinen Anfang zu formalisieren, d. h. den Tatbestand an ein bestimmtes Verfahren zu binden, in dem der Staatsnotstand verkündet wird. Von den untersuchten Notstandsrechten wurde auf ein solches Verfahren im Interesse der Effektivität nur in der WRV völlig verzichtet (Art. 48 Abs. 2 WRV). ${ }^{1 \circ}$ Im Folgenden werden also in diesem Punkt nur das ungarische und das deutsche Notstandsrecht untersucht. Das Grundgesetz sieht für den äußeren Notstand eine formelle Verkündung vor, nicht hingegen für den inneren Notstand (Art. 9I Grundgesetz). Dieser härtere (und für den äußeren Notstand gerade nicht charakteristische) Umgang mit der inneren Gefahr, die sich auf den "Bestand oder die freiheitliche demokratische Grundordnung des Bundes oder eines Landes « bezieht (Art. 91 Abs. I Grundgesetz), ist mit den schlechten Erfahrungen Weimars, d. h. mit der Angst vor den bösen Geistern der Vergangenheit zu erklären. Die Verkündung (bzw. Feststellung) des Staatsnotstandes - wenn es so etwas gibt - erfolgt normalerweise durch die Volksvertretung, und zwar mit einer qualifizierten Mehrheit (Art. I I ja Abs. I Satz 2; Art. 80 a Abs. I Satz 2 Grundgesetz; Art. 9 Abs. 3 lit. g, h und i i. V. m. Art. I9 Abs. 4 ungVerf).

Unter dem Gesichtspunkt der Effektivität kann dies allerdings wegen der Umständlichkeit und dem damit verbundenen Zeitverlust problematisch sein. Deshalb kann diese Feststellung im Fall der Dringlichkeit auch von anderen Organen getroffen werden. Dieses andere Organ ist in Ungarn der Präsident (Art. I9/A Abs. I ungVerf), in Deutschland der Gemeinsame Ausschuss des Bundestages und des Bundesrates (Art. i I sa Abs. 2, Art. 53a Grundgesetz).

Manchmal kann aber sogar dieses einfachere Verfahren zu zögerlich sein, und deshalb - um effektiv den Notstand zu bewältigen - wird ohne irgendwelche Feststellung des Notstandes die Möglichkeit zu sofortigen Maßnahmen vorgesehen. Diese gefährliche Möglichkeit besteht allerdings ausschließlich im Fall eines unerwarteten militärischen Angriffes (Art. I Isa Abs. 4 Grundgesetz; Art. i9/E Abs. I ungVerf).

2. Das Ende des Staatsnotstandes wird eindeutig im Zeichen der Angst geregelt: Furcht davor, dass die zuständigen Organe »vergessen «, den Notstand für beendet zu erklären, um so ihre außerordentliche Macht zu behalten. In diesem Sinne sieht Art. I I 5 l Abs. 2 Satz 2 Grundgesetz eine »unverzügliche « Beendigung des Verteidigungsfalles vor, wenn die Voraussetzungen für seine Feststellung nicht mehr bestehen. Eine ähnliche (allerdings weicher formulierte) Bestimmung ist in Art. I9/A ungVerf zu finden. Mit dem formalen Ende des Notstandes treten automatisch alle notstandsrechtlichen Grundrechtseinschränkungen außer Kraft. ${ }^{\text {I }}$ Da die WRV keinen formalen Anfang des Notstandes kannte, enthielt sie naturgemäß auch keine Bestimmung über sein Ende.

3. Im Zeichen der Verhältnismäßigkeit werden verschiedene Stufen bzw. Arten des Staatsnotstandes bestimmt (Art. 80a, Art. 9i und Art. I I sa Grundgesetz; Art. I9 Abs. 3 lit. g, h und i ungVerf). ${ }^{22}$ In dieser Weise wird nur so viel und sol-

9 Die zutreffende Bestimmung des Tatbestandes bereitet im Allgemeinen nicht unerhebliche Schwierigkeiten, da man gerade solche Situationen vorhersehen müsste, die wegen ihrer Anomalität schwer vorhersehbar und tatbestandlich zu fassen sind, s. Stern (Fn. 8), I 326.

ı Vgl. G. Anschütz, Die Verfassung des Deutschen Reiches vom i r. Aug. I919, Komm., I4. Aufl. I933, Art. 48 Anm. 7 über die »Deformalisierung des Ausnahmerechts«.

I I Vgl. States of Emergency. Their Impact on Human Rights. A study prepared by the International Commission of Jurists, 1983,460 .

I 2 Zur detaillierten Analyse der diesbezüglichen deutschen Rechtslages W. Waleki, Artikel 8oa des Grundgesetzes und Maßnahmen zur Erhöhung der Verteidigungsbereitschaft, I I 98 s. 
che Art von Gewaltenkonzentration zugelassen, die absolut notwendig ist. Die Gefahr des eventuellen Missbrauchs wird also vermindert.

\section{I.2 Organisatorische Regelungen}

Um die für die effektive Bewältigung des Notstandes erforderliche Gewaltenkonzentration durchführen zu können, können neue Organe geschaffen werden. In Art. I9/B Abs. 2 ungVerf ist hierfür ein Verteidigungsrat vorgesehen. Dieser besteht aus dem Präsidenten (zugleich auch Präsident des Verteidigungsrates), aus dem Präsidenten des Parlaments, aus den Fraktionsvorsitzenden, aus dem Premierminister und aus den Ministern. Der Generalstabschef der Armee hat Beratungsrecht. Eine ähnliche Organkreation für Notstandsfälle findet sich weder im Grundgesetz noch gab es sie in der WRV. ${ }^{13}$

Hingegen wurden sowohl in das Grundgesetz als auch in die ungVerf Organbestandsgarantien eingebaut. Diese dienen dazu, Kontrollorgane zu behalten, um den Gefahren der Gewaltenkonzentration entgegenzuwirken. Diese Kontrollorgane sind teilweise die Volksvertretungen (Art. i ish Abs. I Satz I und Abs. 3 Grundgesetz; Art. 28/A Abs. I und 2 ungVerf) und teilweise die Verfassungsgerichte (Art. I I g und Art. I I sh Abs. I Satz 3 Grundgesetz; Art. I9/B Abs. 6 ungVerf). Das Grundgesetz enthält aber auch im Interesse der Effektivität Organbestandsgarantien: Um die Handlungsfähigkeit zu bewahren, wird die Amtszeit des Bundespräsidenten verlängert (Art. I I sh Abs. I Satz 2 Grundgesetz) und das Misstrauensvotum gegen den Bundeskanzler erschwert (Art. I I $\mathrm{h}$ Abs. 2 Grundgesetz).

\section{I.3 Kompetenzregelungen}

Das jeweilige Notstandsrecht schreibt - in erster Linie im Interesse der Effektivität - die normale verfassungsrechtliche Kompetenzordnung um. Dies bedeutet in der Regel eine Zentralisierung.

Dies erfolgt einerseits durch den Übergang von Kompetenzen von einem Organ auf ein anderes Organ. Ein solcher Kompetenzübergang ist überflüssig, wenn das handelnde Organ eine Blankoermächtigung hat (Art. 48 Abs. 2 WRV: »Der Reichspräsident kann« die »nötigen Maßnahmen treffen «, ähnlich Art. 48 Abs. 3 WRV bezüglich der Landesregierungen). ${ }^{4}$ Falls dies aber nicht der Fall ist (Ungarn, BRD), dann müssen die Kompetenzprobleme näher geregelt werden. Im Grundgesetz wird die Gesetzgebungskompetenz des Bundes im äußeren Notstand gegenüber der der Länder verstärkt (Art. I I sc Abs. I und 3 Grundgesetz) bzw. vom Gemeinsamen Ausschuss ausgeübt (Art. I I se Abs. I Grundgesetz). Die Exekutivautonomie der Länder wird praktisch aufgehoben, indem sie der Weisung der Bundesregierung unterstellt werden (Art. 9I Abs. 2 und Art. is f. Abs. I Z 2 Grundgesetz). ${ }^{\text {Is }}$ In diese Zentralisierungslinie passt

I 3 Der Gemeinsame Ausschuss (Art. i s ge Grundgesetz) hat zwar praktisch nur im Verteidigungsfall Funktionen, aber existiert auch im Normalfall.

I4 Detailliert s. D. Esklony, Das Recht des inneren Notstands. Verfassungsgeschichtliche Entwicklung unter besonderer Berücksichtigung der tatbestandlichen Voraussetzungen von Notstandsmaßnahmen und ihrer parlamentarischer Kontrolle, 2000, 88 ff. Deshalb wurde auch über die »Diktaturgewalt des Reichspräsidenten" gesprochen, so z. B. R. Grau, HdbDStR II, 274 m. w. N.

is Vgl. P. Eichhorn, Besondere Formen der Zusammenarbeit von Bund und Ländern im Katastrophenfall und zur Aufrechterhaltung der inneren Sicherheit $1998,54$. 
Art. I I sb Grundgesetz, der anordnet, dass mit der Verkündung des Verteidigungsfalles die Befehls- und Kommandogewalt über die Streitkräfte vom Bundesverteidigungsminister auf den Bundeskanzler übergeht. Eine Erweiterung der Befugnisse der Streitkräfte im Notstand findet in Art. 87a Abs. 3 und 4 Grundgesetz statt, indem die Streitkräfte auch im inneren Bereich des staatlichen Lebens Befugnisse ausüben dürfen (z. B. Schutz von zivilen Objekten). Die einzige Dezentralisierung in der bundesdeutschen notstandsrechtlichen Kompetenzordnung ist für den Fall vorgesehen, dass die Bundesebene nicht imstande ist, die notwendigen Maßnahmen zu treffen. In diesem Fall können die Länder den Bundesgrenzschutz einsetzen (Art. I I 5 i Abs. I Grundgesetz). ${ }^{16}$

In Ungarn werden die Kompetenzen des Präsidenten, der Regierung und teilweise des Parlaments beim oben erwähnten Verteidigungsrat konzentriert (Art. 19/B Abs. 3 ungVerf). Von den Parlamentskompetenzen werden von ihm nur die vom Parlament ausdrücklich übertragenen (Art. I9/B Abs. 3 lit. a ungVerf), ferner die armeebezogenen Parlamentskompetenzen (Art. I9/B Abs. I lit. $a$ und $b$ ungVerf) ausgeübt. Die ungarische Notstandszentralisierung ist also wesentlich stärker (d. h. bei einem einzigen Organ zentralisiert) als die deutsche. Und dieses Organ besteht überwiegend aus Regierungsmitgliedern. Die Gründe für diese sogar übermäßige Konzentration (und somit für die Verabsolutierung der Effektivität) sind mit der der deutschen ähnlichen Regelungsphilosophie (d. h. mit der Dominanz der Furcht vor Missbrauch) schwer zu vereinbaren.

\subsection{Rechtsetzung}

Die jeweiligen Rechtsetzungsverfahren werden in manchen Fällen im Interesse des schnellen Handelns erleichtert. In diesem Sinne sieht Art. I Isd Grundgesetz ein abgekürztes Verfahren bei dringlichen Gesetzesvorlagen im Verteidigungsfall vor. In der WRV oder in der ungVerf findet man ähnliche Regelungen nicht, da die Rechtsetzungskompetenzen bei einem solchen Organ konzentriert sind (Reichspräsident bzw. Verteidigungsrat), dessen Entscheidungsverfahren nicht umständlich ist.

Auch Fragen der Normenhierarchie werden in den Notstandsrechten berührt. Diese ermöglichen den Bruch der Regeln der normalen Normenhierarchie in dieser "abnormalen « Situation (Art. I I sk Abs. I Grundgesetz; Art. I9/B Abs. 4 ungVerf). Allerdings ist der Vorrang der Verfassung (Art. I I s e Abs. 2 Grundgesetz; Art. 19/B Abs. 4 ungVerf) unangetastet, und auch die Geltungsdauer dieser Ausnahmeregelungen ist begrenzt bzw. ihre Verlängerung an die Zustimmung der Volksvertretung gebunden (Art. i isk Abs. 2 und 3 Grundgesetz; Art. I9/B Abs. 5 und Art. I9/C Abs. 4 ungVerf). Ähnliche Grenzen der Notstandsmaßnahmen sind der WRV unbekannt. ${ }^{17}$

\section{I.5 Grundrechtseinschränkungen}

Eine ganz prekäre Frage ist die der Grundrechtseinschränkungen. Im Staatsnotstand sind sie unvermeidlich, aber aus Furcht vor Missbrauch gelten einige 
Grundrechte auch im Notstand uneingeschränkt weiter. Dies kann entweder so geschehen, dass die nicht einschränkbaren Grundrechte taxativ aufgezählt werden (Art. 8 Abs. 4 ungVerf), oder so, dass die einschränkbaren Grundrechte aufgezählt werden (Art. 48 Abs. 2 Satz 2 WRV; Art. I I sc Abs. 2 Grundgesetz). Im Grundgesetz - in einer wenig übersichtlichen Weise ${ }^{18}$ - werden bei den einzelnen Grundrechten (z. B. Art. I I Abs. 2 Grundgesetz) die notstandsspezifischen Fragen der Grundrechtseinschränkung geregelt.

\section{I.6 Kontrolle}

Ein ganz wichtiger Regelungsbereich betrifft schließlich die Kontrolle. Die Kontrolle kann zwar die Effektivität erschweren, ist aber für den Erhalt der Verfassungsstaatlichkeit unentbehrlich.

Diesbezüglich findet man sogar in der notstandsrechtlich sehr lapidaren WRV Vorkehrungen: I. Einerseits soll der Reichspräsident die getroffenen Maßnahmen »unverzüglich « dem Reichstag zur Kenntnis geben (Informationspflicht), 2. andererseits soll er auf Verlangen des Reichstags diese Maßnahmen außer Kraft setzen (Gehorsamspflicht) (Art. 48 Abs. 2 S. 2 und 3 WRV). Eine ähnliche Regelung ist in Art. 87 a Abs. 4 S. 2, Art. 9 I Abs. 2 S. 3 , Art. I I ff. Abs. 2 und Art. i I 5 Abs. I Grundgesetz bzw. in Art. I9/C Abs. 3 ungVerf enthalten. Auch die obengenannten Organbestandsgarantien sind teilweise durch die Notwendigkeit der Kontrolle motiviert.

\section{I.7 Folgerungen}

Wie eben gezeigt wurde, sind die einzelnen Notstandsregeln immer entweder dem Effektivitätsprinzip oder der Furcht vor Missbrauch zuzuordnen. Auch der Unterschied in der Detailliertheit der Regelung ist durch dieses Begriffspaar zu erklären. Im Allgemeinen kann man nämlich feststellen, dass die Notstandsregelungen dort detaillierter sind, wo man mehr Angst vor dem Missbrauch der Notstandsermächtigungen hat(te). ${ }^{19}$ Die Detailliertheit der geltenden deutschen Regelung ist auf die schlechten Erfahrungen in Weimar zurückzuführen, die der im Jahre 1989 eingeführten ungarischen Regelung auf das Schreckensbild des polnischen Beispiels. ${ }^{\circ}$ Dies heißt allerdings nicht, dass die Notstandsregelungen in moderneren Verfassungsstaaten immer detailliert geregelt sind, wie das Beispiel Frankreichs verdeutlicht: Art. I 6 frVerf hat eine dem Art. 48 WRV ähnlich lapidare Formulierung und eine ähnliche Regelungstechnik. Die Entscheidung der einzelnen Rechtsordnungen für die Detailliertheit bzw. für die undetaillierte Effektivität ist immer Ergebnis dieser Rechtsgüterabwägung im Lichte der geschichtlichen Erfahrungen (und der Fähigkeit, daraus zu lernen). ${ }^{2 \mathrm{I}}$ Wie immer bei Rechtsgüterabwägungen (zwischen Effektivität und Normativität), hilft das Verhältnismäßigkeitsprinzip, die richtige Lösung zu finden: sowohl hinsichtlich demokratische und rechtsstaatliche Traditionen fehlen, präzise Regelungen besonders wichtig sind.«

20 Im Dezember 198 I verhängte General Jaruzelski das Kriegsrecht in Polen, um die demokratischen Oppositionsbewegungen unterdrücken zu können,s, States of Emergency (Fn. I I), 86.

2 I Stern (Fn. 8), r.90. 
der Detailliertheit als auch hinsichtlich der Ermächtigungen (Mittel). ${ }^{22}$ Das

heißt, je größer die Störung, desto mehr Mittel (und weniger Hürden) sollten vorgesehen werden. ${ }^{23}$

Diese Rechtsgüterabwägung kann allerdings nur da geschehen, wo die Normativität der Verfassung wirklich ein Rechtsgut ist. Die Institution des Staatsnotstandes macht also nur in einem Verfassungsstaat Sinn. ${ }^{24}$ Deshalb verzichten viele Diktaturen auf solche Regelungen: Dort braucht man keine zusätzlichen Ermächtigungen, da die Staatsmacht sowieso unbegrenzt ist. ${ }^{25}$

Das andere Extrem ist, wo man wegen der Furcht auf notstandsrechtliche Ermächtigungen völlig verzichtet. Für diese irrationale Lösung bietet die Verfassungscharta von Serbien und Montenegro ein Beispiel: Auf Bundesebene ist kein Staatsnotstand vorgesehen. ${ }^{26}$ In diesem Fall hatte die Angst des kleineren Gliedstaates (Montenegro) vor dem Missbrauch (d. h. vor Zentralisierung durch Notstandsmaßnahmen) das Effizienzprinzip völlig besiegt.

\section{Theorien über die Natur des Staatsnotstandes}

Die theoretischen Konstrukte, mit denen der Staatsnotstand erfasst wird, sind in zwei Gruppen aufzuteilen: I. staatszentrierte Theorien und 2. verfassungszentrierte Theorien. ${ }^{27}$

\section{I Staatszentrierte Theorien}

Die Vertreter dieser Gruppe gehen davon aus, dass der Staat eine vorrechtliche (nicht positivrechtliche - also in diesem Sinne naturrechtliche) Befugnis zu selbsterhaltenden Aktionen hat. ${ }^{28}$ Die gemäßigte Version sehen diese Befugnisse als vom positiven Recht verdrängbar an, die klassische Version hingegen nicht.

\section{I.I Der klassische Staatszentrismus}

Der derzeit bekannteste zeitgenössische Vertreter des - auf die vorkonstitutionellen Zeiten zurückgehenden - klassischen Staatszentrismus bezüglich der Natur des Staatsnotstandes ist Klaus Stern. Seiner Ansicht nach hat der Staat immer ein ungeschriebenes, überpositives Notrecht, das durch positives Recht

22 Vgl. Koja (Fn. 19), 65. Zum Zusammenhang von Verhältnismäßigkeit und Abwägung im allgemeine s. $R$. Alexy, Zur Struktur der Rechtsprinzipien, in: B. Schilcher/P. Koller/B-Ch. Funk (Hrsg.), Regeln, Prinzipien und Elemente im System des Rechts, 2000, 35.

23 Hesse, Staatsnotstand (Fn. 6), 202.

${ }_{24}$ Koja (Fn. 19), 65 f.

25 Dementsprechend existierte z. B. in der Originalfassung der ungarischen Verfassung (1949) keine Institution des Staatsnotstandes. Eine stalinistische Verfassung brauchte nicht unbedingt einen Staatsnotstand: der Staat befand sich sowieso im permanenten informellen Staatsnotstand. Zu den vorkonstitutionellen Zeiten ähnlich Stern (Fn. 8), I 297.

26 A. Jakab, Die Verfassungscharta von "Serbien und Montenegro«, ZaöRV 2003/3, 8 I I.

27 Der Vorteil eines ausländischen Autors in diesem empfindlichen theoretischen Bereich ist, dass er schärfer und pointierter formulieren kann, ohne die Diskussionspartner zu verletzen, da er als outsider offensichtlich an keinem innerdeutschen Kampf teilnehmen möchte. In diesem Sinne werde ich versuchen, die Theorielager ehrlich und kontrastiert zu schildern.

28 Nach Koja (Fn. 19), I 2 sind diese Vorstellungen auf die Hegelsche Idee des Staates als präeminente Institution zurückzuführen. Koja hat teilweise Recht, allerdings muss man auch in Betracht ziehen, dass diese Ideen schon vor Hegel bekannt waren, s. E. R. Huber. Deutsche Verassungsgeschichte, Bd. I, I960, 654 . 
Die Argumentation dieser Juristen ist folgende: Die Normativität setzt die Normalität der Verhältnisse voraus. ${ }^{3 \text { I }}$ Das heißt, »dass Normen nur für normale Situationen gelten und dass die vorausgesetzte Normalität der Situation ein positivrechtlicher Bestandteil ihres Geltens « ist. ${ }^{32}$ In Ausnahmesituationen können also Normen den Staat nicht binden. In diesen Ausnahmesituationen hat der Staat hingegen ein Notrecht auf Selbsterhaltung, das wegen der Anormalität der Ausnahmesituation von Normen nicht ausgeschlossen werden kann. 33

Der naturrechtliche Charakter dieser Gedankenführung ist offensichtlich: Es wird dem Staat ein vorpositives Existenzrecht (ius eminens) ${ }^{34}$ eingeräumt. ${ }^{35}$ Und zwar nicht nur neben der Verfassung, sondern auch gegen sie, ${ }^{6} \mathrm{da}$ die Verfassung in einer (per definitionem anormalen) Notsituation nicht gelten kann. Einige sagen es offen, 37 andere ein bisschen zurückhaltender. Offen ist in diesem Sinne Herbert Krüger, nach dem es ausgeschlossen ist, dieses Notrecht überhaupt $\mathrm{zu}$ institutionalisieren. ${ }^{8}{ }^{8}$ Zurückhaltender formuliert (aber im Endeffekt mit demselben Ergebnis) Stern, der zwar nicht offen die Sinnlosigkeit jeglicher Notstandsregelung feststellt, aber ein System der von der jeweiligen Verfassung unabhängigen Schranken der Notstandsbefugnisse ausarbeitet. ${ }^{39}$ Diese Schranken wären I. Schutz essentieller Verfassungsgüter, 2. Notstandsbefugnisse nur als ultima ratio einsetzbar, 3. Abwägung kollidierender Rechtsgüter, 4. Über-

29 Stern (Fn. 8), 1337.

30 C. Schmitt, Die Diktatur. Von den Anfängen des modernen Souveränitätsgedankens bis zum proletarischen Klassenkampf, I92 I, IX. Nur in seiner Formulierung, aber nicht im Wesen, abgewiesen von Stern (Fn. 8), I337. Auch M. Schröder, Staatsrecht an den Grenzen des Rechtsstaates, AöR i978, I 34; G. Flor, Staatsnotstand und rechtliche Bindung, DVBl. 1958, I49; Kirchhof (Fn. 5), 98, 100; U. Scheuner, Der Verfassungsschutz im Bonner Grundgesetz, in: FS Erich Kaufmann, I950, 3 I 8 f.; H. Nawiasky, Allgemeine Staatslehre, Band II/2, I955, I08. Auch so: E. Siegers, Staatsnotrecht. Geschichte, Inhalt und Begründung, 1974, I 25 ff.; H.-E. Folz, Staatsnotstand und Notstandsrecht, I962, I 87 ff.; H. Kurz, Volkssouveränität und Volksrepräsentation, I965, 317; R. Ziblmann, Legitimität und Legalität des Notrechts, I950, 72.

3 I H. Krüger, Allgemeine Staatslehre, 2. Aufl. I966, 3 I; C. Schmitt, Politische Theologie, 2. Aufl. 1934, I9. Dies ist auch von den später zu analysierenden gemäßigten Staatszentrierten anerkannt, s. E.-W. Böckenförde, Der verdrängte Ausnahmezustand, NJW 1978, I884: »Entfällt die vorausgesetzte Normallage, entfällt der Bezugspunkt für die intendierte Regulierungskraft der Norm. «̈̈hnlich H. Heller, Staatslehre, I934, 255.

32 C. Schmitt, Legalität und Legitimität, in: Verfassungsrechtliche Aufsätze aus den Jahren I924-1954 (1958), 32 I.

33 Der Staat wird als prelegale (vorrechtliche) Institution aufgefasst, dessen Gewalt ursprünglich unbegrenzt ist, und nur das Recht ihn zähmt. Vgl. die Schmittianische Sichtweise auch bei dem gemäßigten Staatszentrierten Böckenförde (Fn. 3 I), i 885 . Ähnlich Kirchbof (Fn. 5), i I 7 f.

$34 \mathrm{MwN}$ für die Geschichte des Terminus bei Schröder (Fn. 30), I 32.

35 In diesem Sinne C. F. v. Gerber, Grundzüge des deutschen Staatsrechts, 3. Aufl. 1880, 42 Fn. 2: »In der Anerkennung des Staatsnothrechts liegt der Gedanke, dass es ein Existenzrecht des Staates über sein normales verfassungsmäßiges Leben hinaus giebt, welches in anomalen Nothverhältnissen zur Erscheinung kommt.« E. Kaufmann, Zur Problematik des Volkswillens, I93 I, I4: »Für den äußersten Fall gehört ein letztes Notrecht neben den verfassungsrechtlich normierten und formierten Ausnahmerechten zum ungeschriebenen naturrechtlichen Bestande jedes Verfassungsrechtes. "Ähnlich R. v. Jhering, Der Zweck im Recht, 6-8. Aufl. I923, 330: »Wie der einzelne Mensch, so habe auch der Staat im Falle der Bedrohung seiner Existenz ein solches Notrecht. « Oder aus der Antike: Cicero, De Legibus III, 3: »Salus rei publicae suprema lex esto.«

36 Krüger (Fn. 31), 3 I: »Notrecht impliziert seinem Begriffe nach den Rückgriff auf das natürliche Recht gegen das positive Gesetz." Genauso Stern (Fn. 8), I 336.

37 Z. B. G. Meyer/G. Anschütz, Lehrbuch des deutschen Staatsrechts, 7. Aufl. 1919, 906: "Sicher steht nur eines: die Verfassung will nicht, kann nicht wollen, daß [. . .] das Staatsleben stillsteht. [. . .] Hier hört das Staatsrecht auf; die Frage [. . .] ist keine Rechtsfrage. «Oder eben Schmitt (Fn. 3 I), I I über den Vorrang dieses Existenzrechts gegenüber dem positiven Recht: »Souverän ist, wer über den Ausnahmezustand entscheidet.« Diese Entscheidung und dieser Ausnahmezustand könne vom positiven Recht nicht gebunden werden, s. Schmitt (Fn. 30), IX.

38 Krüger (Fn. 30); 3 I.

39 Die Schranken sollten zeigen, dass es beim ungeschriebenem Notstandsrecht nicht um »offene Generalermächtigung « geht, $S_{3}$ Stern $(\mathrm{Fn}$. 8), 1, 3373. Ausdrücklich gegen den diesbezüglichen Vorwurf von Böckenförde (En. $3 \mathrm{I})$, I $8 \Omega_{3}$. 
maßverbot und 5. die Absicht, in die Normallage zurückzukehren. Für den ersten Blick scheint Stern hier die Argumentation an das positive Verfassungsrecht zu binden (Schutz essentieller Verfassungsgüter als Schranke der Ausübung vorpositiver Notstandsbefugnisse). Dies ist allerdings nur der Schein, da diese Forderung seinerseits nicht näher beleuchtet wird und solche weit interpretierbaren »essentiellen Verfassungsgüter" die Detailregelungen (die das eigentliche Herz der geltenden deutschen Notstandsverfassung sind) nicht ersetzen können. Das positive Verfassungsrecht wird also nicht rehabilitiert, sondern nur seine wichtigsten Prinzipien werden zum Referenzpunkt gemacht.

Nach Stern kann also das positive Recht das (naturrechtliche) Existenzrecht des Staates nicht verdrängen. Dafür bietet er aber ein System von nicht-positivierten Schranken der Ausübung dieses Rechtes. Es bleibt allerdings offen, was für einen Sinn das positive Notstandsrecht dann überhaupt noch hat.

Nach der Ansicht von Klaus Stern sollte es nur dann Geltung beanspruchen, wenn es dem Existenzrecht des Staates nicht widerspricht. Im Rahmen dieses Existenzrechts sei also die Normativität der positiven Notstandsregelungen anzuerkennen - ähnlich wie im Allgemeinen die Normativität des positiven Rechts (»des Gesetzes«) nur innerhalb der Rahmen des Naturrechts (»des Rechts«) anzuerkennen sei.4 $4^{\circ}$ Das - eventuell dem positiven Recht widersprechende - Existenzrecht könne aber weiterhin jederzeit unmittelbar ausgeübt werden. ${ }^{4 \mathrm{I}}$ Die Normativität der verfassungsrechtlichen Notstandsregelungen ist also in dieser Konzeption immer von den vorrechtlichen Notstandsregeln abhängig. Im Ergebnis wird hier die Notstandsverfassung zu einem nicht-justiziablen verwaltungsinternen Aktionsplan (oder zu einer bloßen Richtlinie) degradiert, und zum eigentlichen Notstandsrecht wird das Naturrecht. Wenn nämlich das positiv-verfassungsrechtliche Notstandsrecht (üblicherweise von der Exekutive) verletzt wird, gibt es immer eine "gute juristische Ausrede«, nach der auf der Grundlage der vorpositiven Notstandsbefugnisse die Verletzung des positiven Rechts geschehen sei. Dies macht eine effektive rechtliche Kontrolle unmöglich.

\section{I.2 Die Gemäßigten}

Im Gegensatz zu den vorher genannten klassischen Staatszentrierten gehen die Gemäßigten davon aus, dass das vorpositive Existenzrecht des Staates durch das positive Verfassungsrecht verdrängt werden kann. ${ }^{2}{ }^{2}$ Diese Verdrängung erfolgt durch detaillierte Regelung.

I. Ein bekannter Vertreter dieser Richtung, Ernst-Wolfgang Böckenförde, sieht im ungeschriebenen Notrecht eine »offene Generalermächtigung « für jedes staatliche Organ, deren einzige Begrenzung die Verhältnismäßigkeit wäre, nicht aber eine Begrenzung nach Träger, Umfang und Reichweite. Eine solche offene Generalermächtigung verstoße gegen die Grundstruktur einer rechtsstaatlichen 
Verfassung.43 Gemäß Böckenförde ist aber die geltende deutsche Notstandsregelung nicht detailliert genug, um die überverfassungsrechtlichen Ausnahmebefugnisse zu ersetzen. ${ }^{44}$. Im Endergebnis ist er also dem klassischen Staatszentrismus sehr nahe - obwohl er seine staatstheoretische Grundlegung angreift. Nach ihm gelte nämlich in Deutschland auch derzeit überpositives Notstandsrecht, und man müsste gerade darum eine detailliertere Notstandsregelung schaffen, damit es verdrängt wird.

2. Andere folgen hingegen eher dem Willen des Verfassunggebers, nach dem mit der im Jahre 1968 eingeführten Notstandsregelungen die Ausübung der (ansonsten im Hintergrund existierenden) überverfassungsrechtlichen Befugnisse ausgeschlossen ist. 45

\subsection{Verfassungszentrierte Theorien}

Die verfassungszentrierten Theorien erkennen die Existenz vorrechtlicher staatlicher Befugnisse nicht an. Als Ausgangspunkt der Argumentationen dient hier immer die positive (gesetzte) Verfassung.

\subsection{Die klassische Version}

Nach der klassischen (radikalen) Version dieser Ansicht sind Staatsnotbefugnisse nur diejenigen, die in der Notstandsverfassung ausdrücklich als solche genannt sind. ${ }^{6}$

Diese Verfassungszentrierten betonen die Gefahr des Missbrauchs ungeschriebener und damit unlimitierter Notstandsermächtigungen stärker als die Staatszentrierten.47 Ganz einleuchtend formuliert diesen Standpunkt Adolf Arndt: »Alles Spekulieren mit einem >überverfassungsgesetzlichen Notstand als einer Erlaubnis zu Maßnahmen, die nicht von der urkundlichen Verfassung gerecht-

43 Böckenförde (Fn. 31), I 883.

$44 \mathrm{Zu}$ seinem de lege ferenda Regelungstext s. E.-W. Böckenförde, Ausnahmerecht und demokratischer Rechtsstaat, in: FS Martin Hirsch, I98 1, 259 ff., insb. $264 \mathrm{ff.}$

45 Danach sei Ziel der Notstandverfassungsgebung im Jahre 1968 gerade, »den Rückgriff auf ungeschriebene Verfassungsgrundsätze durch ausdrückliche Regelungen zu erübrigen.« s. Schrift. Bericht des Rechtsauschusses, BT-Drucks. V/2873. Zitiert von Stern (Fn. 8), I 329.

46 R. Thoma, HdbDStR II, 232: "Ein Staatsnotrecht über das verfassungsrechtlich regulierte hinaus kann es im geordneten republikanischen Verfassungsstaat nicht geben, so wie es auch schon im Kaiserreich und selbst in den konstitutionellen Monarchien (deren Struktur immerhin Zweifel erlaubte) nicht gegolten hatte." A. Hamann, Zur Frage des Ausnahme- oder Staatsnotrechts, DVBl. 1958, 405 ff.; R. Speidel, Der Begriff der Staatsnotstandslagen und die Möglichkeiten ihrer Abwehr in der Bundesrepublik Deutschland, I964, 94 ff.; A. Voigt, Ungeschriebenes Verfassungsrecht, VVDStRL 1952, 44; A. Arndt, Der Rechtsstaat und sein polizeilicher Verfassungsschutz, NJW 1961, 900; H. M. Parche, Der Einsatz von Streitkräften im inneren Notstand. Zugleich ein Beitrag zur Lehre vom rechtsstaatlichen Handeln der Exekutive und seiner Kontrolle, Diss. Münster, 1974, 3 und 179; Möllers (Fn. 2), 267; H. Oberrenter, Notstand und Demokratie, I978, 89 ff., und insb. I I 3, I 20 f.; W. Kägi, Verfassung als rechtliche Grundordnung des Staates, I945, I 18; M. Krenzler, An den Grenzen der Notstandsverfassung. Ausnahmezustand und Staatsnotrecht im Verfassungssystem des Grundgesetzes, 1974, 74; Wehrs, F.-B. v., Zur Anwendbarkeit des Notstandsrechts der Bundesrepublik Deutschland, Diss. Mainz, i 97 I, i I 6 f. Überraschenderweise ist die Ansicht von K. Hesse, Die normative Kraft der Verfassung, 1959, 24 und ders., Grundzüge (Fn. 6), 300 ff. dem Kelsenschen eigentlich ähnlich. Nach ihm könne man sich nicht auf überpositives Notstandsrecht berufen. Man müsse allerdings auch sehen, dass im Falle der Nichtregelung des Notstandes die staatlichen Organe doch so tun würden.

47 Daneben wird auch selbst die naturrechtiche Einstellung als unwissenschaftlich kritisiert, s. Koja (Fn. 4), 398 und ders. (Fnerig), ro.rch IP '172.22.53.54', am 26.04.2023, 15:14:23. 
fertigt werden, ist nichts als eine verwerfliche Beschönigung des Verfassungs-

bruchs, des Verfassungsverrats. $\ll 48$

Der Staatsnotstand wird also nicht als außerverfassungsrechtliche Lage, sondern als verfassungsrechtliche lex specialis aufgefasst. 49

Das Argument des dem Verfassungsrecht überlegenen Existenzrechts des Staates wird abgelehnt. ${ }^{\circ}$ Eine andere enthüllende Formulierung stammt von Kelsen: »Hinter der treuherzigen Versicherung, dass der Staat ,leben' müsse, verbirgt sich meist nur der rücksichtslose Wille, dass der Staat so leben müsse, wie es diejenigen für richtig halten, die sich zur Rechtfertigung eines ,Staatsnotrechts' bedienen. « ${ }^{\mathrm{I}}$

Dies heißt allerdings nicht, dass nach der Meinung dieser Gruppe jeder Bruch des Notstandsrechts - selbst wenn der Bruch im Interesse der Wiederherstellung des verfassungsmäßigen Zustandes geschieht - gemäß der positiven Vorschriften bestraft werden sollte. Nachträglich könne nämlich ein solcher Bruch durch Indemnitätsbeschluss des zuständigen Organs, etwa des Parlaments gebilligt werden. Der Bruch als solcher könne aber nicht geleugnet werden..$^{2}$ In dieser Konzeption kann zwar eine moralische Verpflichtung zur Rechtsverletzung (im Interesse der Rettung des Verfassungsstaates) vorhanden sein; dies ist aber streng von der juristischen Beurteilung des Falles zu unterscheiden.

\subsubsection{Die offene Version}

Eine offenere verfassungszentrierte Version wird von Eckart Klein vertreten, nach der zwar alle staatlichen Notstandsbefugnisse auf die positivrechtliche Verfassung zurückführbar sein müssen - diese Befugnisse können allerdings auch implizite Befugnisse sein, d. h. nicht ausdrücklich genannte, da die Verfassung auch die allgemeine Pflicht enthält, sie zu erhalten.53 Das grundsätzliche Problem dieser Auffassung ist, dass sie - nur scheinbar auf der positiven Verfassung basierend - eine Ansicht vertritt, die dem Willen des Verfassunggebers widerspricht. Der Wille des Verfassunggebers war nämlich im Jahre I 968 gerade der Ausschluss der ungeschriebenen Notstandsbefugnisse. 54

48 A. Arndt, Demokratie - Wertsystem des Rechts, in: ders., Notstandsgesetze - aber wie? Köln, I962, I 3. Genauso J. Barthélemy/P. Duez, Traité de Droit Constitutionnel, 1933, 242 darüber, dass überpositives Notstandsrecht kein Rechtsgrundsatz, sondern nur »une théorie politique que le gouvernement pourra invoquer devant le parlement pour expliquer son illégalité et la rendre politiquement excusable« ist. Ähnlich G. Jellinek, Allgemeine Staatslehre, 3. Aufl. 1929, 359 darüber, dass man »um eklatante Verletzungen der Staatsordnung zu beschönigen « die Kategorie des Staatsnotrechts entwickelt und angewendet habe, »die doch nur ein anderer Ausdruck für den Satz ist, dass Macht vor Recht geht. "Oder eben A. Merkl, Allgemeines Verwaltungsrecht, I927, I66: überpositives Notstandsrecht als die »letzte Rückzugsposition der Verfechter einer teilweisen gesetzlichen Ungebundenheit der Verwaltung.«

49 Koja (Fn. 4), 399. S. noch ders. (Fn. I9), I 4: »Der so verstandene Staatsnotstand ist also - dies ist wichtig - ein Verfassungszustand und kein Zustand der Verfassungslosigkeit «

so A. Arndt, Der Rechtsstaat und sein polizeilicher Verfassungsschutz, NJW 1961, 899: "Ein Verfassungsstaat hat keine andere ,Raison' als seine Verfassung. « Ähnlich K. Hesse, Ausnahmezustand und Grundgesetz, DÖV I955, 74I.

5 I H. Kelsen, Allgemeine Staatslehre, I925, i 57.

ऽ2 Koja (Fn. I9), I7.

53 E. Klein, Der innere Notstand, HdBStR VII, I992, 4I 2. Ähnlich die amerikanische Doktrin der implied powers, s. K. Doehring, Das Staatsnotrecht in den Vereinigten Staaten von Amerika, in: Das Staatsnotrecht in Belgien, Frankreich, Großbritannien, Italien, den Niederlanden, der Schweiz und den Vereinigten Staaten von Amerika, I955, 2 I 2 ff.

54 S. oben Fn. 45 . 
Die verschiedenen Ansichten lassen sich folgendermaßen darstellen. Das eine ordnende Kriterium ist die theoretische Basis, d. h. die Anerkennung vorpositiver Notstandsbefugnisse. Die Verfassungszentrierten leugnen diese Befugnisse, die Staatszentrierten hingegen bejahen sie. Das andere ordnende Kriterium ist praktischer - nämlich ob gemäß den jeweiligen Autoren in der geltenden bundesdeutschen Verfassungslage auch im Grundgesetz nicht ausdrücklich erwähnte Notstandsbefugnisse gegeben sind. Danach können wir zwischen Textgebundenen und Nicht-Textgebundenen unterscheiden.5s

\begin{tabular}{|c|c|c|}
\hline $\begin{array}{l}\text { existieren vorpositive } \\
\text { Notstandsbefug- } \\
\text { nisse? (theo- } \\
\text { retische } \\
\text { Basis) } \\
\text { sind } \\
\text { nur die im } \\
\text { Grundgesetz } \\
\text { ausdrücklich } \\
\text { erwähnten Not- } \\
\text { standsbefugnisse } \\
\text { auszuüben? (prak- } \\
\text { tische Seite) }\end{array}$ & $\begin{array}{c}\text { ja } \\
\text { (Staatszentrierte) }\end{array}$ & $\begin{array}{c}\text { nein } \\
\text { (Verfassungszentrierte) }\end{array}$ \\
\hline ja (Textgebundene) & $\begin{array}{c}\text { Maunz, Zippelius, } \\
\text { Doehring, Dürig, } \\
\text { Trotter }\end{array}$ & $\begin{array}{c}\text { Hesse, Möllers, Wehrs, } \\
\text { Krenzler, Oberrenter, } \\
\text { Parche } \\
\text { (Kägi, Arndt, Duez, } \\
\text { Barthélemy, Hamann, } \\
\text { Voigt, Speidel, } \\
\text { G. Jellinek, Koja, } \\
\text { Kelsen, Merkl) }\end{array}$ \\
\hline $\begin{array}{l}\text { nein (nicht Text- } \\
\text { gebundene) }\end{array}$ & $\begin{array}{l}\text { Böckenförde, Stern, } \\
\text { Schröder, Kirchhof, } \\
\text { Siegers } \\
\text { (Schmitt, Krüger, Flor, } \\
\text { Jhering, Zihlmann, } \\
\text { Meyer, Anschütz, } \\
\text { Gerber, Kaufmann, } \\
\text { Scheuner, Kurz, } \\
\text { Nawiasky, Folz) }\end{array}$ & E. Klein \\
\hline
\end{tabular}

Den Gefahren des Missbrauchs wird die verfassungszentrierte und textgebundene Lösung mit der moralischen Ergänzung (s. o.) am ehesten gerecht, ohne Effizienzprobleme aufzuweisen - deshalb steht sie auch der Auffassung des Autors dieser Zeilen am nächsten. 
Abschließend möchte ich aber noch einen anderen, einen unüblich zynischen Lösungsversuch zeigen. Zynisch ist er in dem Sinne, dass er praktisch auf die juristische Argumentation verzichtet und damit die vorherigen Überlegungen stark relativiert. Er stellt aber klar, dass die Übernahme der dargestellten Argumentationsmuster stark rechtskulturabhängig und somit dem üblichen rechtswissenschaftlichen Diskurs nicht zugänglich ist. Die folgende Gedankenführung wurde von der Rechtstheorie von Herbert Hart inspiriert. ${ }^{6}$

Gemäß Hart hängt die Rolle des Naturrechts von der jeweiligen Rechtsordnung ab: In manchen Rechtsordnungen betrachten Juristen Naturrecht als Teil der Rechtsordnung (z. B. USA), in anderen hingegen nicht. Und einen besseren Test als die Anerkennung der Juristen kann man nicht bekommen (rule of recognition). Ähnliches gilt für die Textgebundenheit: Wenn die Juristen einer Rechtsordnung bei einer bestimmten Problematik üblicherweise textgebunden argumentieren, dann ist dies dort das Recht - wenn sie sich hingegen auf naturrechtliche Normen berufen, gehört auch dieses Naturrecht zum Recht.57 Zugespitzt könnte man formulieren: Recht ist die herrschende Meinung der Juristen..$^{8}$

Wenn wir auf die Tabelle sehen, 59 gibt es auf den ersten Blick keine eindeutig herrschende Meinung bezüglich des deutschen Notstandsrechts. ${ }^{60}$ Allerdings kann Hart uns doch weiterhelfen. Wenn wir nämlich die Fragen (I. Gibt es vorpositive Notstandsbefugnisse?, 2. sind nur die im Grundgesetz ausdrücklich erwähnten Notstandsbefugnisse auszuüben?) im Einzelnen untersuchen, dann finden wir für beide Fragen als h. M. zu bezeichnende Antworten. Die überwiegende Mehrheit der Autoren befürwortet nämlich die Existenz vorpositiver Notstandsbefugnisse, und gleichzeitig die überwiegende Mehrheit der Autoren (diese Mehrheit besteht teilweise aus anderen Autoren) denkt aber, dass nur die im Grundgesetz ausdrücklich erwähnten Notstandsbefugnisse auszuüben sind wenn auch für diese überwiegenden Meinungen andere Autoren stehen. Wenn man also mit Hart die genannten Fragen beantworten möchte, dann kann man sagen: In der deutschen Rechtsordnung gibt es zwar vorpositive Notstandsbefugnisse, aber derzeit können sie nicht ausgeübt werden, weil sie vom positiven Verfassungsrecht verdrängt wurden.

56 H. Hart, The Concept of Law, 2. Aufl. 1994, I00 ff. insb. I09.

57 Hart (Fn. 56), 204.

58 Dies heißt allerdings nicht, dass nach dieser Konzeption die jeweilige h. M. unangreifbar wäre. Wenn nämlich die bisher nicht erkannte implizite Folge einer h. M. ,A' der h. M. ,B' widerspricht, dann kann man die eine h. M. angreifen (und zwar diejenige, die gemäß der h. M. ,C' wichtiger ist).

59 Es zählen nur die kursiv gedruckten Namen.

60 Für die Lösung der ungarischen Lage ist er aber vom Nutzen, denn da ist der textgebundene Verfassungszentrismus vorherrschend, s. implizit z. B. Zs. Balogh/A. Holló/I. Kukorelli/J. Sári, Az Alkotmány magyarázata [Verfassungskommentar], 2003, 309 ff.; A. Úffalvi, "Szükség törvényt bont « avagy a rendkívüli jogrend szabályozása a Negyedik Köztársaságban [»Not kennt kein Gebot « oder die Regelung der Ausnahmerechtsordnung in der Vierten (Ungarischen) Republik], Magyar Közigazgatás, I990, 6I 4 ff.; A. Jakab, A jogszabálytan fobb kérdéseirel [Hauptragen einer Theorie- der normativen Rechtsakte], 2003 , I 2 ff. 
I Das Grunddilemma des Notstandsrechts ist, ein Gleichgewicht zwischen Effektivität der Bekämpfung der staats- bzw. verfassungsgefährdenden Situation und den Garantien des Verfassungsstaates zu finden.

2. Die verschiedenen Regelungen und die Unterschiede in der Regelungsdichte sind mit diesem Dilemma zu erklären.

3. Je größer die (regelmäßig auf geschichtliche Erfahrung basierende) Angst vor Missbrauch, desto detaillierter ist eine Notstandsverfassung.

4. Das eigentliche Ziel des Notstandsrechtes sollte sein, den Rückweg in den »normalen « Verfassungszustand zu sichern. Deshalb macht die Institution des Staatsnotstandes nur da einen Sinn, wo der Gewaltausübung verfassungsrechtliche Schranken auferlegt sind (Verfassungsstaat).

6. Die theoretischen Konstrukte, mit denen der Staatsnotstand erfasst werden, sind in zwei Gruppen zu teilen: (a) staatszentrierte Theorien, und (b) verfassungszentrierte Theorien.

7. Die staatszentrierten Theorien erkennen ein vorpositives Existenzrecht des Staates an, die verfassungszentrierten hingegen nicht.

8. Von dieser Frage ist zu unterscheiden, ob die Argumentation der jeweiligen Autoren textgebunden ist, also ob sie außer den in der jeweiligen Verfassung ausdrücklich erwähnten Notstandsbefugnissen auch andere Notstandsbefugnisse anerkennen oder nicht.

9. Eine allgemeine Antwort auf die Frage, welcher der Autoren Recht hat, kann nicht gegeben werden. Die Frage ist nämlich auf zwei Ebenen zu beantworten: (a) wessen Argumente sind stärker (danach scheinen die textgebundenen Verfassungszentrierten am besten gelegen zu sein), und (b) welche Ansicht hat mehr Anhänger. Die Antwort auf die zweite Frage hängt immer von der herrschenden Meinung der Juristen der jeweiligen Rechtsordnung ab. In der deutschen Rechtsordnung gibt es nach der h. M. zwar vorpositive Notstandsbefugnisse, aber derzeit können sie nicht ausgeübt werden, weil sie vom positiven Verfassungsrecht verdrängt wurden. 